Received: April 27, 2017

Accepted: May 10, 2017

Published: June 13, 2017

\title{
An Insight into the Capability of Composite Technology to Enable Magnesium to Spread its Wings in Engineering and Biomedical Applications
}

Nature has various ways to inspire humans through a lot of its creations that researchers in different disciplines are trying to understand and mimic in order to enhance current state of technology and quality of life. From materials perspective, nature uses composites in its creations involving plants and animals with bone as a classical example.

Magnesium is one of the nature's most favorite metallic element with its wide spread presence in planet earth and the whole universe besides in human body (fourth most abundant cation). It was ignored to a large extent so far and that ignorance is, in part, responsible for the worsening climate and contamination of land mass and water bodies all over the globe.

The key attributes of magnesium is its light weight ( $\sim 35 \%$ lighter than aluminum), abundance, non-toxicity (a nutritional element for both plants and humans) and recyclability. These attributes ensures reduction in carbon di oxide emissions, sustainability and enhanced health of living organisms. As magnesium is non-toxic, its recyclability is not an issue and it will not need special disposal methods or infrastructure. Besides these attributes, over last 15 years the price of magnesium is showing a downward trend making it an increasingly cost effective option with every passing day for weight critical applications.

In view of the sustainability of technology, non-toxicity and reducing negative climate changes (such as global warming), magnesium based materials provide a perfect alternative to replace aluminum based materials and steels in many movable/mobile engineering applications to reduce fuel consumption and in biomedical applications to avoid revision surgery, patient trauma and doctor's time. It may be noted that magnesium is involved in more than 300 chemical reactions in body and enhances the performance of muscles, nerves, cardiovascular system, immune system and sleep pattern. On the other hand aluminum is neurotoxic and can lead to cognitive deficiency, dementia, adverse effect on central nervous system and reproductive system.
Current materials selectors have a number of options to match different steels and aluminum based alloys with various end applications. For immovable applications such as in infrastructure, steels are most favored while in movable applications requiring a source of energy such as in transportation sector, aluminum based alloys are chosen widely. This can be attributed to decades of research to develop these materials and availability of supporting technologies and skilled manpower related to these materials. The decade of 1980 has seen, for example, replacement of steels in transportation sector (dominated by automobile and aerospace) with aluminum based materials ( $\sim 35 \%$ density of steels) greatly assisting in reducing fuel consumption. However with a progressive rise in population and the number of vehicles on roads, climate took a severe beating (global warming) in last 2 decades that was not seen before. This has resulted in rivers changing courses, icebergs appearing in towns of Canada, excessive and unpredictable rains leading to economic losses equivalent to billions of dollars. With transportation sector contributing to $\sim 31 \%$ of total CO2 emissions, researchers are looking into ways to realize further weight savings in transportation sector.

To address the issue of light weighting, magnesium based materials are a perfect choice, however, the number of options available to material selectors in terms of commercially available alloys and the range of properties that they exhibit is rather limited when compared to aluminum based materials. To address this limitation, researchers worldwide over last 1 decade are trying to develop new alloys particularly focusing on rare earth (RE) elements as alloying elements in magnesium. The limitations of using rare earth elements are their limited availability, high cost and toxicity. Besides the compositional adjustment, the properties of magnesium and its alloys can be stretched using the composite technology. Composite technology, in principal, involves the judicious use of a reinforcing phase to enhance either general or targeted properties of the base materials. 
Most of the research activities on magnesium based composites initiated in 1990s with the initial prime focus on micron size reinforcement and to some extent on interconnected reinforcements. Micron size reinforcements clearly enhanced hardness, strength, elastic modulus, damping and tribological response of magnesium while adversely affecting ductility and energy absorption capability during plastic deformation. This was primarily attributed to the activation of particle based damage mechanisms under application of load. Use of interconnected reinforcement was another unique approach which led to increase in elastic modulus of composite, at times beyond the prediction of rule of mixture. The limitation of micron length scale reinforcement (poor end ductility and toughness) was addressed by the judicious selection of reinforcement in nano-length scale. The use of nano-length scale reinforcement minimizes the possibility and probability of particle damage mechanisms (particle cracking and debonding) leading to an enhancement in tensile ductility and tensile strength simultaneously. Another significant role of certain reinforcements in nano-length scale is their ability to change the texture of magnesium which has a significant influence on its ductility and corrosion under wet conditions. Ceramic reinforcements belonging to carbide, oxide, nitride and boride families were commonly investigated along with carbon based reinforcements such as carbon nanotubes (SWCNT and MWCNT) and graphene (platelets and sheet). These reinforcements enhanced different properties to different extents depending primarily on their type and amount. However, research work conducted so far on magnesium based nanocomposites has established the capability of nano reinforcement to enhance ambient and high temperature strength, ductility, creep resistance, wear resistance, ignition resistance, fatigue life, dynamic response, oxidation and wet corrosion properties.

Attempts were also made in last decade to develop magnesium composites containing amorphous/metastable reinforcements. These reinforcements exhibit physical size in micron length scale while the microstructure remains amorphous or in nano length scale. Being metallic in nature these reinforcements exhibit good bonding with magnesium and particularly for amorphous reinforcement their high elastic limit and strength ensures minimal cracking under the application of load. As a result this group of composites exhibit superior hardness, damping and compressive response. Exploration of these composites is still limited and needs further attention from international research community.

Yet another group of magnesium based composites currently being investigated are syntactic composites containing hollow reinforcement. Attempts have been made to incorporate cenospheres (hollow fly ash particles) which is an industrial waste in magnesium. Initial results have established that use of such particles can easily bring down the density of magnesium from 1.74 $\mathrm{g} / \mathrm{cc}$ to $\sim 1.5 \mathrm{~g} / \mathrm{cc}$ which is closer to that of plastics. In addition, presence of cenospheres significantly enhances hardness, compressive strength and ductility. Syntactic composites such as the one containing cenospheres, in addition, can also reduce the cost of end material and the final product as cenospheres are available at insignificant cost. Moreover, the use of cenospheres besides advancing the properties of magnesium also addresses the waste management issue related to fly ash particles.

In a nutshell, magnesium technology in general is a promising technology which will be certainly adopted in a significant way in very near future and in both engineering and biomedical applications. It has a strong and clear potential to give a better life style to our future generation while simultaneously assisting in reversing the ongoing climate changes.

\section{Biography}

Dr Manoj Gupta is a former Head of Materials Division of the Mechanical Engineering Department and Director designate of Materials Science and Engineering Initiative at NUS, Singapore. He did his Ph.D. (Materials Science) from University of California, Irvine, USA (1992), and postdoctoral research at University of Alberta, Canada (1992). To his credit are: (i) 'Disintegrated Melt Deposition' technique, a unique liquid-state processing method, and (ii) 'Hybrid Microwave Sintering' technique, an energy efficient solid-state processing method, to synthesize $\mathrm{Al}$ and $\mathrm{Mg}$ light-metal alloys/micro/nano-composites. He has published over 440 peer reviewed journal papers and owns two US patents. He has also co-authored five books and serves as Chief Editor and editorial boards of many international journals. A multiple award recipient, Dr Gupta was invited by many universities in countries like India, Japan, Saudi Arabia and France as visiting researcher/professor. 